\title{
Is vitamin A consumption a risk factor for osteoporotic fracture?
}

\author{
Margo E. Barker ${ }^{1 *}$ and Aubrey Blumsohn ${ }^{2}$ \\ Human Nutrition Unit ${ }^{l}$ and Bone Metabolism Unit ${ }^{2}$, Division of Clinical Sciences (North), \\ University of Sheffield, Northern General Hospital, Herries Road, Sheffield S5 7AU, UK
}

\begin{abstract}
Severe vitamin A toxicity is known to have adverse effects on skeletal health. Studies involving animal models and case reports have documented that hypervitaminosis $\mathrm{A}$ is associated with bone resorption, hypercalcaemia and bone abnormalities. More recently, some epidemiological studies have suggested that high habitual intake of vitamin A could contribute to low bone mineral content and fracture risk. The evidence relating to the possible deleterious role of vitamin A in bone health is of variable quality and is potentially confounded by collinearity of nutrient intake and difficulties in assessing vitamin A exposure. Furthermore, because intake of vitamin A varies between studies it is not possible to define an intake threshold associated with harm.
\end{abstract}

Vitamin A intake: Hypervitaminosis A: Bone health: Osteoporotic fracture risk

Insufficient supply of a wide variety of nutrients such as $\mathrm{Ca}$ and $\mathrm{Mg}$ may affect bone health. However, excessive intake of a few nutrients, such as protein or $\mathrm{Na}$ may also be associated with skeletal disease (Dawson-Hughes, 2003; Harrington \& Cashman, 2003). There is also some evidence that excessive intake of vitamin A could contribute to low bone mineral content and fracture risk. The evidence relating to the possible deleterious role of vitamin $\mathrm{A}$ in bone health is of variable quality and is subject to several possible confounding influences.

Elisha Kent Kane, an eminent Victorian Arctic explorer and surgeon, first noted deleterious effects of markedly excessive intake of vitamin A. He recorded in his journal that his crew became drowsy, depressed and confused after eating polar bear liver. These effects were probably psychotic symptoms in response to vitamin A excess (Goodman, 1995).

Numerous animal studies have shown that very excessive intake of vitamin A can cause ossification of cartilage, increased bone resorption, extraosseous calcification, hypercalcaemia, suppressed parathyroid hormone levels, and fractures (Moore \& Sharman, 1979; Frankel et al. 1986; Hough et al. 1988). The doses in these studies were exceptionally high $(>2100 \mu \mathrm{g}$ retinol equivalents per $\mathrm{kg} / \mathrm{d})$. Case reports in human subjects have also documented increased bone resorption, hypercalcaemia, skeletal pain and radiographic changes in chronic vitamin A poisoning (Frame et al. 1974). The doses consumed in these cases was of the order of 15 $000-30000 \mu \mathrm{g}$ retinol equivalents/d for several years, and at least ten times higher than typical habitual dietary intake. In some cases supplementary vitamin A was taken as a prophylactic for acne or as an aid to improve night vision. Children and adolescents seem to be particularly susceptible to vitamin A toxicity (Frame et al. 1974). Synthetic retinoids used to treat skin conditions such as acne may also result in skeletal disease in some patients (Milstone \& Leachman, 2001).

\section{Vitamin A metabolism and the skeleton}

The liver stores $90 \%$ of body vitamin A as retinyl esters, and this reserve is sufficient to maintain vitamin A supplies for several months. Vitamin A is transported in serum as retinol bound to retinol-binding protein. Decreased plasma retinol is likely to reflect vitamin A insufficiency. However, plasma retinyl esters are thought to be more sensitive as an indicator of vitamin A toxicity (Smith \& Goodman, 1976). It is possible that lipoprotein-associated retinol has greater cellular toxicity than retinol-binding protein-complexed retinol (Smith \& Goodman, 1976).

Carotenoids, or so-called 'provitamin A' (in particular $\beta$-carotene), are converted to retinol in the mucosal cells of the small intestine by carotene dioxygenase. A commonlyused rule of thumb is that $6 \mu \mathrm{g} \beta$-carotene in mixed foods is nutritionally equivalent to $1 \mu \mathrm{g}$ retinol (Department of Health, 1991), but other conversion factors have been suggested. Studies have shown that the efficiency of the conversion of provitamin $\mathrm{A}$ to vitamin $\mathrm{A}$ is less than 
previously thought (West et al. 2002). The US Institute of Medicine (2000) has suggested a conversion factor of $12 \mu \mathrm{g}$ $\beta$-carotene/retinol equivalent. There are no reports of vitamin A toxicity from ingestion of excessive amounts of carotenoids (Bendich \& Langseth, 1989).

The precise mechanism by which vitamin A might influence skeletal health is unclear (Anderson, 2002; Denke, 2002; Rohde \& Deluca, 2003). At a cellular level, retinoic acid is the active metabolite. Retinol is released in target cells and converted to retinoic acid, which is likely to exert its effect by binding to specific nuclear receptors. These receptors are members of the retinoic acid receptor or retinoid X receptor nuclear transcription family, and are found in nearly every cell, including osteoblasts, osteoclasts and chondrocytes. Retinoic acid suppresses osteoblast activity and stimulates osteoclast formation in vitro. In some instances the apparent toxic effects of vitamin A might be related to altered vitamin $\mathrm{D}$ metabolism, concurrent ingestion of substantial amounts of vitamin $\mathrm{D}$ or binding of vitamin A to receptor heterodimers. Animal studies (Clark \& Bassett, 1962; Metz et al. 1985; Rohde et al. 1999; Anderson, 2002) and in vitro studies have variously reported antagonistic or synergistic interactions between these two vitamins in terms of their skeletal effect. In human studies ingestion of $15 \mathrm{mg}$ retinyl palmitate (corresponding to one serving of liver) has been reported to reduce the intestinal response to vitamin D (Johannson \& Melhus, 2001). Another study reported that stimulation of bone resorption by retinoic acid was independent of vitamin D (Rohde \& DeLuca, 2003).

\section{The problem of assessing vitamin A status}

The majority of vitamin A is preformed (as retinol) in the diet. Proformed vitamin A ( $\beta$-carotene and other carotenoids) comprises about $30 \%$ of the total vitamin A intake. However, dietary retinol intake is difficult to assess. Retinol intake is largely derived from a small subset of foods such as liver, dairy products, eggs and oily fish. Some foods, such as margarine, may also be fortified with vitamin A. The main food sources of carotenoids are dark leafy green vegetables, yellow and orange vegetables and fruit.

It is possible to assess vitamin A exposure by dietary assessment or to measure retinol status using biomarkers. As vitamin $\mathrm{A}$ is found in relatively few foods day-to-day variation in intake is substantial (Willett, 1998) and assessment of intake using short-term diet records is likely to be of no value. It has been estimated that dietary records would have to be kept for a minimum of 15 weeks (Willett, 1998) in order to estimate long-term intake of vitamin A with any extent of accuracy. In dietary surveys it is rare for recording to be carried out for $>7 \mathrm{~d}$ and this timescale will inevitably lead to substantial misclassification. Retrospective food-frequency questionnaires may circumvent the problem of timescale, but are highly dependent on the accuracy with which intake of specific foods (such as liver) are recalled.

It is also difficult to assess vitamin A intake from supplements. Most multivitamin preparations contain vitamin A, and the amount of retinol varies between brands. Whilst most multivitamin supplements in the UK contain $800 \mu \mathrm{g}$ vitamin A, some supplements may contain more than double this amount. There is substantial heterogeneity in reported supplement use between studies. In North America multivitamins contributed $41 \%$ of the intake of preformed retinol in the 1994 round of data collection in the Nurse's Health Study (Feskanich et al. 2002). However, the last national survey of British women in 1987 (Gregory et al. 1990) reported that only $4 \%$ of preformed retinol intake was derived from supplements. Supplement use within the USA also varies; about one-quarter of adults took supplements containing vitamin A in the National Health and Nutritional Examination Survey (NHANES III; Ballew et al. 2001) compared with $53 \%$ of women in the Nurse's Health Study cohort (Feskanich et al. 2002). Surveys in Scandinavian countries report widespread use of cod liver oil, and $40 \%$ of retinol intake may be supplied in this form (Sigurdsson et al. 2001).

As the liver stores $>90 \%$ of the vitamin A, assessment of status would ideally involve measurement of retinol in liver tissue. However, this approach is clearly impractical in epidemiological studies. Plasma retinol may not necessarily reflect subtle differences in vitamin A status, since the liver buffers blood levels over a wide range of normal dietary intake over the short term (Willett, 1998). Administration of $7500 \mu \mathrm{g}$ retinyl palmitate/d for 8 weeks has no appreciable effect on plasma retinol (Willett et al. 1983), although a 3 -month period of supplementation may result in a small increase (Willett, 1998). Retinol-binding protein is a negative acute-phase protein, and plasma retinol decreases in the presence of infection. This effect was shown clearly in NHANES III, in which increased serum C-reactive protein was associated with low plasma retinol (Stephensen \& Gildengorin, 2000). This relationship may confound studies because chronic inflammatory disease may be associated with skeletal disease. Retinyl esters usually comprise $<5 \%$ of the total serum vitamin A. However, if vitamin A intake is excessive serum retinyl esters are markedly increased. Retinyl esters:total serum retinol may be a good marker of vitamin A excess (Willettt, 1998). However, there are few data on the time-course of response to altered retinol intake. Laboratory measurements are also subject to inaccuracy and retinol assays may vary widely between laboratories (Hulshof et al. 2002).

\section{The problem of geography and comparability between studies}

Several recent studies purport to show that vitamin A intake at the upper end of the population distribution is related and unrelated to skeletal health. These studies have been carried out in different countries that have differing dietary intakes, food fortification practices and use of supplements. Dietary sources of vitamin A vary by geography. Margarine is fortified with vitamin A in the UK. In other countries such as the USA and Sweden vitamin A may also have been added to food products such as milk and breakfast cereals. The upper end of the distribution of intake in one population may be very different from that in another population, and these studies are, therefore, unlikely to be comparable. This variation in intake distribution may, in part, explain these differing results. Furthermore, laboratory methods and 
techniques of dietary assessment are also unlikely to be comparable.

Table 1 shows dietary intake distributions of total retinol (carotenoids and preformed retinol) in various populations. Although Scandinavian populations are reputed to ingest very high amounts of vitamin A, a study of Swedish women (Melhus et al. 1998) reported intakes close to that of British women (Gregory et al. 1990). However, the mean intake of these populations is about five-eighths that of the mean daily intake reported in the USA in the Nurse's Health Study (Feskanich et al. 2002) and in an Icelandic study (Sigurdsson et al. 2001). Even within the USA intake distributions from surveys differ. In the Nurse's Health Study the top quintile of average intake of retinol from food was at the 90th percentile of similarly-aged women in NHANES III and the bottom quintile corresponded to the 50th percentile of women in NHANES III (Denke, 2002).

In the UK the reference nutrient intake for vitamin $A$ is set at $700 \mu \mathrm{g}$ retinol equivalents/d for men and $600 \mu \mathrm{g}$ retinol equivalents/d for women (Department of Health, 1991). Recorded intakes are generally about 2.5 times higher. Whilst the North American dietary reference intakes are slightly higher, mean intakes are still a factor of three times the recommended dietary allowance, and a substantial proportion of women in the Nurse's Health Study (Feskanich et al. 2002) recorded an intake of vitamin A $>3000 \mu \mathrm{g} / \mathrm{d}$.

\section{The problem of collinearity of nutrient intake}

Vitamin A intake is collinear with intake of several other nutrients and lifestyle factors that may influence skeletal health. For example, the correlation with dietary vitamin $\mathrm{D}$ intake was approximately $r 0.88$ in one Icelandic study (Sigurdsson et al. 2000), and the Nurse's Health Study (Feskanich et al. 2002) reported a similar correlation ( $r$ 0.76). Individuals with high vitamin A intake tend to have higher $\mathrm{Ca}$, protein and vitamin $\mathrm{K}$ intakes and a lower alcohol intake, are less likely to smoke, are more physically active and take multivitamin, vitamin $\mathrm{A}$ or $\beta$-carotene supplements (Feskanich et al. 2002). In the Nurse's Health Study $67 \%$ of those in the highest quintile of vitamin A intake took a multivitamin supplement compared with $17 \%$ in the lowest quintile. Adequate statistical correction for these confounders may not be possible.

\section{Design of studies relating vitamin A exposure to skeletal risk}

Studies may be categorised by (1) study design, (2) study end point and (3) how vitamin A exposure was assessed. In terms of study design there are cross-sectional studies, cohort studies and intervention studies, the latter providing the strongest evidence. Studies may also be classified by study end point. Retrospective occurrence of fracture, or measurement of surrogate predictors of fracture risk such as bone turnover or bone mineral density (BMD), may provide useful information. Prospective studies of fracture incidence provide the strongest evidence. Finally, vitamin A status may be assessed using measures of dietary intake or biochemical measurements.

\section{Cross-sectional studies}

Four cross-sectional studies have explored the relationship between vitamin A status and bone mass (Table 2). An early study (Melhus et al. 1998) investigated the association between dietary intake of vitamin A and BMD at various skeletal sites in a community-based sample of 175 Swedish women over a very wide age-range (28-74 years). Preformed retinol intake was assessed over only $28 \mathrm{~d}$ using diet records. There was no significant effect of retinol intake on BMD at intakes of $\leq 500 \mu \mathrm{g} / \mathrm{d}$. However, women with exceptionally high intakes $(>1500 \mu \mathrm{g} / \mathrm{d}$; approximately $5 \%$ of the population studied) had a significantly lower BMD (10\% lower at the femoral neck, $P=0.05)$ in comparison with those with low intakes $(<500 \mu \mathrm{g}$ retinol $/ \mathrm{d})$.

An Icelandic study of 70-year-old women (Sigurdsson et al. 2001) related total vitamin A intake (retinol equivalents) to BMD. Vitamin A intake was assessed using a 130-item food-frequency questionnaire targeted at vitamin Acontaining foods. Total retinol intake ranged from 300 to $7000 \mu \mathrm{g}$ retinol equivalents/d, but a substantial percentage of intake (30) was related to consumption of cod liver oil. No association between intake of vitamin A and BMD was found. The authors hypothesised that the observed lack of effect may be a consequence of the high extent of collinearity ( $r 0.88)$ between intake of vitamin A and vitamin D.

In another cross-sectional study of 250 North American post-menopausal women using serum retinol as an index of exposure there was no association with radial BMD or fracture history (Sowers \& Wallace, 1990). There was also no association between supplement use and radial bone mass or fracture history.

The largest cross-sectional study was that of Ballew et al. (2001). Approximately 6000 North American adults participating in the NHANES III survey had femoral neck and total hip BMD measured in relation to a biomarker of vitamin A exposure (plasma retinyl esters). Retinyl esters were expressed in relation to total serum retinol, with the cut-off point for toxicity regarded as being $>10 \%$. Although about one-third of subjects had elevated serum retinyl esters, no association was observed with BMD.

Table 1. Total retinoid intake ( $\mu \mathrm{g}$ retinol equivalents/d) distributions from food and supplements

\begin{tabular}{lllll}
\hline Study population & Mean & Range & Dietary instrument & Reference \\
\hline Swedish women* & 1510 & $280-7180$ & 28d records & Melhus et al. (1998) \\
British women & 1488 & $250-6326$ & $7 \mathrm{~d}$ records & Gregory et al. (1990) \\
US female nurses & 2373 & $505-8959$ & Food-frequency questionnaire & Feskanich et al. (2002) \\
Icelandic women & 2500 & $400-7500$ & Food-frequency questionnaire & Sigurdsson et al. (2001) \\
\hline
\end{tabular}

${ }^{*}$ Calculated as the sum of retinol and $\beta$-carotene intake. 


\section{Cohort studies}

There have been four cohort studies (Table 3) that have explored the relationship between vitamin A exposure and risk of osteoporosis. The Rancho Bernardo Study (Promislow et al. 2002) assessed the vitamin A intake of $>1000$ elderly Californian (USA) men and women using a food-frequency questionnaire. Vitamin A intake was assessed as preformed retinol from food and supplements, but excluded intake of proformed retinol. In the cohort $50 \%$ of the women and $39 \%$ of the men used supplements containing vitamin A. It was concluded that there is an 'inverse 'U'-shaped' relationship between retinol intake and BMD change, and that supplement use affects the relationship between retinol intake and BMD. For example, amongst female supplement users a higher retinol intake was associated with a lower bone mass and greater bone loss over 4 years, whilst among female non-supplement users a high retinol intake was associated with a greater bone mass and lower bone loss. However, this relationship was reported at only one skeletal site (the femoral neck), was observed only in women and was of borderline statistical significance.

Data from the prospective Nurse's Health Study (Feskanich et al. 2002), involving 72337 post-menopausal women and 18 years of follow-up, support a detrimental effect of retinol intake on hip fracture risk. Total and preformed retinol from the diet and supplements were estimated using food-frequency questionnaires on five occasions throughout the study. There was an increase in the risk of hip fracture in $48 \%$ of women in the highest quintile of intake of total retinol from either food or supplements ( $>3000 \mu \mathrm{g}$ retinol equivalents/d) compared with women in the lowest quintile of intake $(<1250 \mu \mathrm{g}$ retinol equivalents/d). The risk estimates were adjusted for selected confounding dietary factors (intakes of $\mathrm{Ca}$, protein, vitamin $\mathrm{D}$, vitamin $\mathrm{K}$, caffeine and alcohol), as well as selected osteoporosis risk factors. When preformed retinol intake from food and supplements was used as the exposure, the multivariate relative risk was 1.89 (95\% CI 1.33, 2.68) for those in the highest quintile of preformed retinol intake $(>2000 \mu \mathrm{g} / \mathrm{d})$ compared with women who had a low retinol intake $(<500 \mu \mathrm{g} / \mathrm{d})$. When supplement users were excluded the multivariate risk estimate for preformed retinol from food was 1.69 (95\% CI 1.05, 2.74) for the women in the highest quintile of preformed retinol intake $(>1000 \mu \mathrm{g} / \mathrm{d}) v$. those in the lowest quintile of intake $(<400 \mu \mathrm{g} / \mathrm{d})$. Intake of carotenoids showed no association with risk.

A Swedish research group explored the relationship between dietary intake of retinol and risk of hip fracture using a nested case-control design (Melhus et al. 1998). Cases were women aged 40-68 years who had a hip fracture within 5 years of enrolment. Each case was matched with four controls and the intake of preformed retinol was assessed by food-frequency questionnaire. Mean intake of preformed retinol was $960 \mu \mathrm{g} / \mathrm{d}$ in cases and $880 \mu \mathrm{g} / \mathrm{d}$ in controls. It was estimated that there was a two-fold increase in odds for hip fracture in women whose dietary intake of retinol was $>1500 \mu \mathrm{g} / \mathrm{d}$ compared with those consuming $\leq 500 \mu \mathrm{g}$ retinol/d.

In a further cohort study of 2322 Swedish middle-aged men (Michaelsson et al. 2003) fracture risk was assessed in relation to serum retinol and $\beta$-carotene levels measured at baseline. Fractures were documented in 266 men during the 30 -year follow-up period. There was a $64 \%$ increase in risk of any fracture and a $250 \%$ increase in risk of hip fracture when the top quintile of serum retinol was compared with the middle quintile. However, the number of men experiencing a hip fracture was very small (only ten in the middle quintile). For every $1 \mathrm{SD}$ increase in serum retinol there was a $26 \%$ increase in the risk of fracture. Serum $\beta$-carotene was not associated with fracture.

\section{Intervention studies}

There are no prospective placebo-controlled studies of supplemental vitamin A intake and fracture risk. Kawaraha

Table 2. Summary of cross-sectional vitamin A studies of vitamin A exposure in relation to skeletal health

\begin{tabular}{llcc}
\hline Study & Assessment & End point & Population \\
\hline Melhus et al. (1998) & Diet intake & BMD & Women \\
Sigurdsson et al. (2001) & Diet intake & BMD & Post-menopausal women \\
Sowers \& Wallace (1990) & Serum retinol & BMD & Post-menopausal women \\
Ballew et al. (2001) & Serum retinyl esters & BMD & Men and women \\
\hline
\end{tabular}

BMD, bone mineral density; Effect, greater BMD.

Table 3. Summary of cohort studies of vitamin A exposure and skeletal health

\begin{tabular}{lllll}
\hline Study & Assessment & End point & Population & Effect \\
\hline Melhus et al. (1998) & Diet & Hip fracture & Post-menopausal women & Yes \\
Promislow et al. (2002) & Diet & BMD and BMD change & Older men and women & Yes with supplements \\
& & & Effect opposite with food \\
Feskanich et al. (2002) & Diet & Hip fracture & Post-menopausal women & Yes \\
Michaelsson et al. (2003) & Serum retinol & All fractures and hip fracture & Men & Yes \\
\hline
\end{tabular}

BMD, bone mineral density; Effect, increased incidence of fracture or greater BMD loss. 
et al. (2002) assessed change in bone turnover in response to supplemental retinyl palmitate $(7565 \mu \mathrm{g} / \mathrm{d}$ for 6 weeks $)$ in men. There was no change in bone turnover, as assessed using one marker of bone resorption (serum N-terminal telopeptides of type I collagen) and two markers of bone formation (bone alkaline phosphatase and osteocalcin). However, the response of other markers of bone resorption was not assessed, and it is not clear whether the duration of supplementation was sufficient to alter body stores of retinol.

\section{Conclusions}

Cross-sectional studies of vitamin A exposure and BMD have generally shown no association between vitamin A status or intake and skeletal risk. A few other studies with more persuasive end points have suggested that excess vitamin A exposure may be a risk factor for fracture. However, all studies are potentially confounded by collinearity of nutrient intake and difficulties in assessing vitamin A exposure. Furthermore, because the intake of vitamin A varies between countries and between studies it has not yet been possible to define an intake threshold associated with harm.

\section{References}

Anderson JJB (2002) Oversupplementation of vitamin A and osteoporotic fractures in the elderly: To supplement or not to supplement with vitamin A. Journal of Bone and Mineral Research 17, 1359-1362.

Ballew C, Galuska D \& Gillespie C (2001) High serum retinyl esters are not associated with reduced bone mineral density in the third National Health and Nutrition Examination Survey, 1988-1994. Journal of Bone and Mineral Research 16, 2306-2312.

Bendich A \& Langseth L (1989) Safety of vitamin A. American Journal of Clinical Nutrition 49, 358-371.

Clarke I \& Bassett AL (1962) The amelioration of hypervitaminosis $\mathrm{D}$ in rats with vitamin A. Journal of Experimental Medicine 115, 147-155.

Dawson-Hughes B (2003) Interaction of dietary calcium and protein in bone health in humans. Journal of Nutrition 133, $852 \mathrm{~S}-854 \mathrm{~S}$.

Denke MD (2002) Dietary retinol - a double-edged sword. Journal of the American Medical Association 287, 102-104.

Department of Health (1991) Dietary Reference Values for Food Energy and Nutrients for the United Kingdom. Report on Health and Social Subjects no 41. London: H.M. Stationery Office.

Feskanich D, Singh V, Willett WC \& Colditz GA (2002) Vitamin A intake and hip fractures among postmenopausal women. Journal of the American Medical Association 287, $47-54$.

Frame B, Jackson CE, Reynolds WA \& Umphrey JE (1974) Hypercalcemia and skeletal effects in chronic hypervitaminosis A. Annals of Internal Medicine 80, 44-48.

Frankel TL, Seshadri MS, McDowall DB \& Cornish CJ (1986) Hypervitaminosis A and calcium-regulating hormones in the rat. Journal of Nutrition 116, 578-587.

Goodman AB (1995) Chromosomal locations and modes of action of genes of the retinoid (vitamin A) system support their involvement in the etiology of schizophrenia. American Journal of Medical Genetics 60, 335-348.
Gregory J, Foster K, Tyler H \& Wiseman M (1990) The Dietary and Nutritional Survey of British Adults. London: H.M. Stationery Office.

Harrington M \& Cashman KD (2003) High salt intake appears to increase bone resorption in postmenopausal women but high potassium intake ameliorates this adverse effect. Nutrition Reviews 61, 179-163.

Hough S, Avioli LV, Muir H, Gelderblom D, Jenkins G, Kurasi H, Slatopolsky E, Bergfeld MA \& Teitelbaum SL (1988) Effects of hypervitaminosis $\mathrm{A}$ on the bone and mineral metabolism of the rat. Endocrinology 122, 2933-2966.

Hulshof P, Brouwer JT, Burema J \& West CE (2002) Bias and random error in retinol measurements of laboratories in countries with populations with mild to severe vitamin A deficiency. Clinical Chemistry 48, 2061-2063.

Institute of Medicine (2000) Dietary Reference Intakes for Vitamin A, Vitamin K, Arsenic, Boron, Chromium, Copper, Iodine, Iron, Manganese, Molybdenum, Nickel, Silicon, Vanadium, and Zinc. Washington DC: National Academy Press.

Johansson S \& Melhus H (2001) Vitamin A antagonizes calcium response to vitamin D in man. Journal of Bone and Mineral Research 16, 1899-1905.

Kawahara TN, Krueger DC, Engelke JA, Harke JM \& Binkley NC (2002) Short-term vitamin A supplementation does not affect bone turnover in men. Journal of Nutrition 132, 1169-1172.

Melhus H, Michaelsson K, Kindmark A, Bergstrom R, Holmberg L, Mallmin H, Wolk A \& Ljunghall S (1998) Excessive dietary intake of vitamin $\mathrm{A}$ is associated with reduced bone mineral density and increased risk of hip fracture. Annals of Internal Medicine 129, 770-778.

Metz AL, Walser MM \& Olson WG (1985) The interaction of dietary vitamin A and vitamin D related to skeletal development in the turkey poult. Journal of Nutrition 115, 929-935.

Michaelsson MD, Lithell H, Vessby B \& Melhus H (2003) Serum retinol levels and risk of fracture. New England Journal of Medicine 348, 287-294.

Milstone LM \& Leachman SA (2001) Do retinoids cause osteoporosis? In Nutritional Aspects of Osteoporosis, pp. 285-293 [P Burckhardt, B Dawson-Hughes and RP Heaney, editors]. New York: Academic Press.

Moore T \& Sharman IM (1979) Hypervitaminosis A combined with calcium deficiency in rats. International Journal of Vitamin and Nutrition Research 49, 14-20.

Promislow JHE, Goodman-Gruen D, Slymen DJ \& Barrett-Connor E (2002) Retinol intake and bone mineral density in the elderly: the Rancho Bernardo Study. Journal of Bone and Mineral Research 17, 1349-1358.

Rohde CM \& DeLuca H (2003) Bone resorption activity of all-trans retinoic acid is independent of vitamin $\mathrm{D}$ in rats. Journal of Nutrition 133, 777-783.

Rohde CM, Puno CWE, Manatt M, Clagett-Dame M \& Deluca HF (1999) Vitamin A antagonises the action of vitamin D in rats. Journal of Nutrition 129, 2246-2250.

Sigurdsson G, Franzson L, Thorgeirsdottir H \& Steingrimsdottir L (2001) A lack of association between excessive dietary intake of vitamin A and bone mineral density in seventy-year-old Icelandic women. In Nutritional Aspects of Osteoporosis, pp. 295-302 [P Burckhardt, B Dawson-Hughes and RP Heaney, editors]. New York: Academic Press.

Smith FR \& Goodman DS (1976) Vitamin A transport in human vitamin A toxicity. New England Journal of Medicine 294, 805-808.

Sowers MF \& Wallace RB (1990) Retinol, supplemental vitamin A and bone status. Journal of Clinical Epidemiology 43, 693-699.

Stephensen CB \& Gildengorin G (2000) Serum retinol, the acute phase response, and the apparent misclassification of vitamin A status in the third National Health and Nutrition 
Examination Survey. American Journal of Clinical Nutrition 72, 1170-1178.

West CE, Eilander A \& van Lieshout M (2002) Consequences of revised estimates of carotenoid bioefficacy for dietary control of vitamin A deficiency in developing countries. Journal of Nutrition 132, 2920S-2926S.
Willett W (1998) Nutritional Epidemiology, 2nd ed. New York: Oxford University Press.

Willett WC, Stampfer MJ, Underwood BA, Taylor JO \& Hennekens CH (1983) Vitamins A, E, and carotene: Effects of supplementation on their plasma levels. American Journal of Clinical Nutrition 38, 559-566. 